\title{
A Hybrid BCI for Enhanced Control of a Telepresence Robot
}

\author{
Tom Carlson, Luca Tonin, Serafeim Perdikis, Robert Leeb, and José del R. Millán
}

\begin{abstract}
Motor-disabled end users have successfully driven a telepresence robot in a complex environment using a BrainComputer Interface (BCI). However, to facilitate the interaction aspect that underpins the notion of telepresence, users must be able to voluntarily and reliably stop the robot at any moment, not just drive from point to point. In this work, we propose to exploit the user's residual muscular activity to provide a fast and reliable control channel, which can start/stop the telepresence robot at any moment. Our preliminary results show that not only does this hybrid approach increase the accuracy, but it also helps to reduce the workload and was the preferred control paradigm of all the participants.
\end{abstract}

\section{INTRODUCTION}

One of the core areas targeted by brain-computer interfaces (BCIs) is neuroprosthetics. This means being able to control robotic and prosthetic devices in order to perform activities of daily living, using brain signals alone. The eventual aim is to empower people with severe motordisabilities to (re-)gain a degree of independence. One of the most significant challenges currently faced is that in addition to high accuracy in the decoding of mental commands, fast decision-making and split attention are critical [1], [2], [3]. There have been several demonstrations of such braincontrolled devices, ranging from robotic arms [4], [5], to hand orthoses [6], [7]; and from telepresence robots [1], [8], to wheelchairs [9], [10], [11]. These works predominantly take spontaneous approaches, where the subjects learn to voluntarily modulate their sensorimotor brain activity. Such a paradigm has proven to be an intuitive and natural manner in which to control neuroprosthetic devices and, after training, motor-disabled end-users have been able to attain levels of performance that are comparable to healthy subjects [8].

Electroencephalogram (EEG), being non-invasive, is a convenient, safe, and inexpensive method that promises to bring BCI technology to people with severe motorimpairments. However, the inherent properties of EEG signals cause EEG-based BCIs to be limited by low information transfer rates. Nevertheless, complex robotic devices have been successfully and reliably controlled by such BCIs, by exploiting smart interaction designs, such as shared control [12], [13], [14], [15]. Millán's group has pioneered the use of shared control in neuroprosthetics, by taking the continuous estimation of the user's intentions and providing appropriate assistance to execute tasks safely and reliably [1], [2], [16].

This research is supported by the European ICT Programme Project FP7224631 (TOBI) and the Swiss National Science Foundation through the National Centre of Competence in Research (NCCR) Robotics.

T. Carlson, L. Tonin, S. Perdikis, R. Leeb and J.d.R. Millán are with the Chair in Non-Invasive Brain-Machine Interface, Center for Neuroprosthetics, School of Engineering, École Polytechnique Fédérale de Lausanne, Lausanne, Switzerland, tom.carlson@epfl.ch

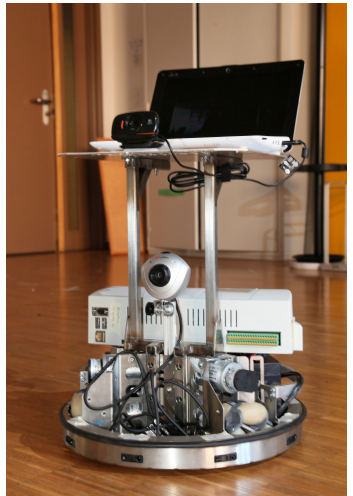

Fig. 1: Our telepresence platform is based upon Robotino by FESTO, which we have equipped with an additional wideangle webcam and a laptop to facilitate remote interactions.

Furthermore, thanks to the mutual learning approach, where the user and the BCI are coupled together and adapt to each other, end-users are able to learn to operate brain-actuated devices relatively quickly (typically in a matter of hours spread across few days [8], [3], [17], [18]).

Previously, we have shown how a two-class motor imagery-based BCI can enable motor-disabled end users to successfully drive a telepresence robot in a complex environment [8], [17]. To date, these users have relied upon an assistant to start and stop the BCI feedback that allows them to drive the robot. Furthermore, they were only able to stop the robot when the shared control system determined that they wished to dock to a particular target. Then, to remain stationary, they had to actively not deliver any BCI commands (intentional non-control), which has proven to be demanding (high workload). However, it is an important element of any telepresence system to be able to reliably start and stop the robot at any point [19]. As a solution, we propose to exploit the hybrid $\mathrm{BCI}(\mathrm{hBCI})$ principle [20], whereby a complementary EMG channel is added to the EEG-based BCI [21] to enable the user to start/stop the robot and (un-)pause the delivery of BCI commands at any moment.

We will briefly describe our telepresence robot platform, explain our motor imagery-based BCI and the additional complementary EMG system. Then we will give the details of our experiment protocol, which investigates the efficacy of the hybrid approach. Finally we will discuss our conclusions that the hybrid approach not only brings benefits in terms of increasing precision, but it also reduces the workload. We will finish by considering the implications of transferring 
such a hybrid approach to severely motor-disabled end users and applying it to other assistive technologies.

\section{METHODS}

\section{A. The Telepresence Platform}

Our telepresence robot is driven using a two-class asynchronous sensory-motor rhythm-based BCI. The robotic platform (see Fig. 1) consists of a commercial circular mobile robot base, Robotino by FESTO, which is equipped with nine proximity sensors. We have added a webcam and a laptop to enhance the environmental perception capabilities and provide the telepresence functionalities [8]. The user can control the motion of the robot by voluntarily delivering one of the two classes (turn left or turn right), or decide not to issue a turning command at all, which yields an implicit third class known as intentional non-control. In this third case, the robot continues with its default behaviour, which is to move forward and avoid obstacles where necessary. This is achieved by a system known as shared control, which takes the environmental context into account when interpreting the user's commands. It decides exactly when and how far to turn, and whether to avoid or dock to the obstacles it encounters along the way [8], [16].

\section{B. The Brain-Computer Interface}

Since the user will perform motor imagery tasks to drive the robot, we acquire monopolar EEG at a rate of $512 \mathrm{~Hz}$ (bandpass filtered between $0.5-100 \mathrm{~Hz}$ ), from 16 electrodes placed over the motor cortex, which are spaced according to the international 10/20 system. We apply a Laplacian spatial filter and then estimate the power spectral density (PSD) over the last second, in the band $4-48 \mathrm{~Hz}$ with a $2 \mathrm{~Hz}$ resolution [1]. We compute the PSD features every $62.5 \mathrm{~ms}$ using the Welch method with 5 overlapped (25\%) Hanning windows of $500 \mathrm{~ms}$. We use canonical variate analysis (CVA) [22] to select the subject-specific features that best reflect the motor-imagery task for each subject and use these to train a Guassian classifier [18]. Evidence about the executed task is accumulated using an exponential smoothing probability integration framework [23]. This helps to prevent commands from being delivered accidentally and enables the user to deliberately not deliver a command in the process previously described as intentional non-control.

\section{The EMG Interface}

In this pilot study, we measure the bipolar EMG of the extensor carpi radialus longus in healthy subjects. This was chosen due to the ease of electrode placement: the muscle is easily identified when extending the wrist (lifting the hand), c.f. Fig. 2. The EMG is acquired in parallel with the EEG, but this time, we bandpass filter the signal between 30$40 \mathrm{~Hz}$. Due to the comparably high signal-to-noise ratio (SNR), we were able to detect the EMG commands by simply applying a subject-specific threshold to the envelope of the signal. The threshold is determined as the midpoint between mean active and mean rest values from the offline training dataset [21]. When transferring the technology to

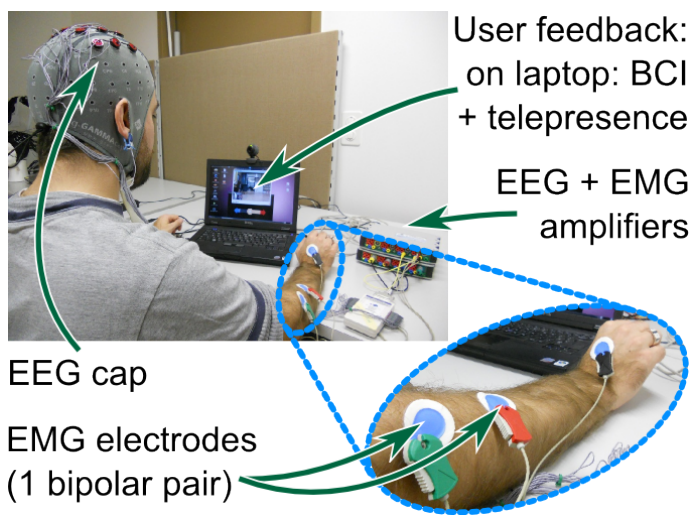

Fig. 2: The hybrid experiment setup. The user performs motor imagination tasks to drive the telepresence robot and extends the wrist to start/stop the robot.

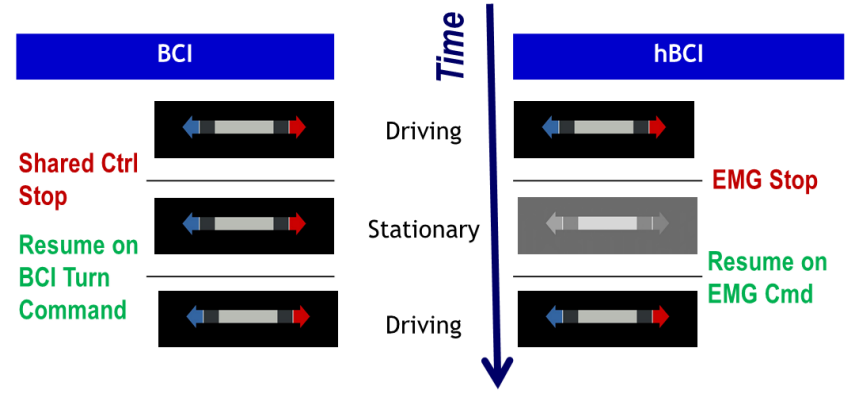

Fig. 3: A comparison between the two control paradigms. On the left is the original BCI protocol (INC condition) and the new $\mathrm{hBCI}$ protocol is on the right (hybrid condition).

motor-disabled end users, we will involve clinician in the EMG electrode placement, such that we target the most appropriate residual voluntary muscular control available, as we have already successfully done in our previous studies with a text-entry system [21]. It is important to note that the EMG interface is used infrequently compared with the EEG interface. This is because motor-disabled end-users often find it fatiguing to voluntarily produce sustained EMG activity from their residual muscular capabilities (e.g. continuous control for sustained periods), yet are able to reliably produce short bursts of EMG activity.

\section{The Control Paradigms}

In the original paradigm, subjects use the BCI to continuously control the robot, which will only stop when its shared control system detects an "obstacle" that is immediately in front of it, such that it is ambiguous as to which direction it should turn to avoid the object. To remain stationary in such a case, users must (intentionally) not deliver any commands, until they are ready to move on (see Fig. 3). This can be extremely demanding, both in terms of manoeuvring the robot precisely to the target location and then deliberately not delivering any more commands, especially if the user wishes to simultaneously interact with someone (telepresence) whilst the robot is stationary. 


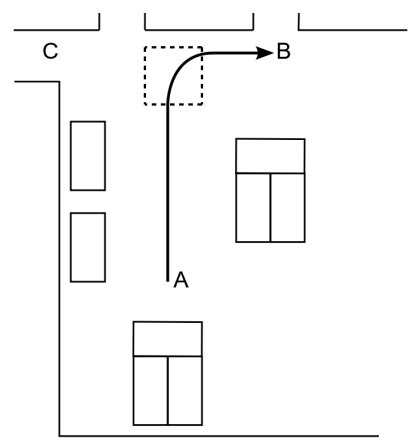

Fig. 4: One of the trajectories $(\mathrm{A} \rightarrow \mathrm{B})$ that the participants had to drive is shown. They also had to drive $\mathrm{B} \rightarrow \mathrm{A}, \mathrm{A} \rightarrow \mathrm{C}$ and $\mathrm{C} \rightarrow \mathrm{A}$. On each trajectory they were instructed to pause just before entering the dotted square region (i.e. before making the turn) and again when they reached the target.

Conversely, in the new hBCI paradigm (see Fig. 3), an additional EMG channel acts as an asynchronous toggle switch, which can start or stop the robot's motion and simultaneously (un-)pause the delivery of BCI commands. In this case, subjects continuously control the motion of the robot using the BCI, until they deliver an EMG command. At which point, the robot stops and the BCI feedback becomes greyed out. The user continues to receive feedback about the status of the BCI, but any commands that are delivered are not sent to the robot. The only way to reactivate the robot and continue driving using the BCI is to send the same EMG command once more. The robot will then begin moving again and the BCI feedback bars will simultaneously become reilluminated in colour, indicating to the user that the robot will now respond to any BCI commands that they might deliver.

\section{E. The Experiment Protocol}

We perform a feasibility study with four healthy males aged $28 \pm 6$ years. They were experienced users of the original BCI telepresence platform, but were naïve to the goals of this experiment. All were all able to reach a BCI accuracy of greater than $90 \%$ in an online cued protocol, prior to undertaking the experiment with the robot. The task involved driving the robot along eight trajectories, each $\sim 7 \mathrm{~m}$ in length (Fig. 4). The subject was audibly cued by the experimenter to pause and resume driving twice at predefined locations along each path. We compare the original BCI control paradigm (condition $I N C$ ) - where the shared controller stops the robot and any BCI command resumes the motion-with the new hBCI paradigm (condition Hybrid), for both short (10 s) and long $(30 \mathrm{~s})$ pauses. The turn directions and pauses were interleaved and counterbalanced within subjects, whereas the control paradigms were block-counterbalanced between subjects.

\section{EXPERIMENTAL RESULTS}

Although we do not yet have enough participants to give any statistically significant results, this pilot study does suggest some noteworthy trends. As can be seen in Fig. 5, it was much more difficult for participants to make the robot remain stationary for a precise period of time using intentional non-control, compared with using the hybrid BCI. This is especially the case for long trials (Fig. 5b), where participants were instructed to stop for a period of 30 seconds, but on average could only remain stationary for around 20 seconds, before they accidentally delivered a command. Furthermore, the variance of the INC trials is greater than that of the hybrid trials, which again highlights the difficulty of precise timing for BCI command delivery [17]. In the hybrid condition, for one of the long pause trials, subject $\mathrm{s} 2$ relaxed and accidentally flexed his arm, which resulted in a false positive in the EMG, thus contributing to the larger negative mean error. It is easy, though, to recover from such an error, since the user need only deliver another EMG command immediately afterwards to stop the robot again.

Importantly, the subjects report a reduction in the perceived task workload, when using the hybrid approach, as can be seen in Fig. 5c. The only task load index (NASA TLX) [24] indicator that showed a tendency to increase, was that of physical demand, which is to be expected, since in the INC paradigm, no physical movements were required. Moreover, all four subjects reported that they preferred to use the hybrid version for stopping, rather than relying upon the shared control to identify a potential location in which to stop the robot and then actively not deliver any commands, in order to remain stationary. This was for two reasons: first, it gave the user more authority over the robot and second it required less effort.

\section{CONCLUSIONS}

This pilot study suggests that not only does the hybrid approach provide a reliable and precise stopping mechanism, but that users are able to successfully complete the task with a lower perceived workload. These results may be transferred to representative end-users, who are able to produce reliable EMG activity (fatigue should be negligible given the sporadic nature of stop commands). The new hybrid control paradigm empowers users to start and stop the BCI controlled robot, without having to rely upon an assistant. This, combined with the lower overall workload, is likely to enable BCI users to work independently for prolonged periods of time.

In the near future, the same technique could be applied to other related devices, such as brain-controlled wheelchairs. Although studies have shown that users were able to successfully complete realistic driving tasks without the hybrid approach [9], [17], the extra control channel increases the user's level of authority and independence, without compromising on safety or impacting heavily on the task load.

\section{ACKNOWLEDGEMENT}

The authors would especially like to thank Marco Creatura and Vasiliki Liakoni for their invaluable help with running some of the experiments, as well as all the volunteer participants for their time and effort. This work is supported by the European ICT Project TOBI (FP7-224631) and the Swiss National Science Foundation through the NCCR Robotics. 


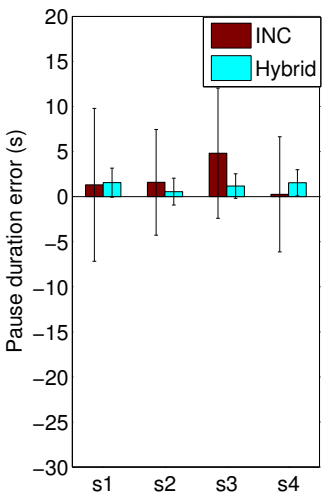

(a) Short (10s) pause trials

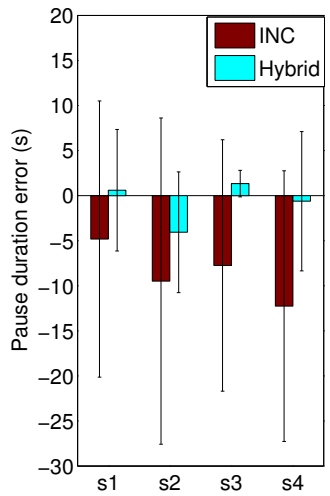

(b) Long (30s) pause trials

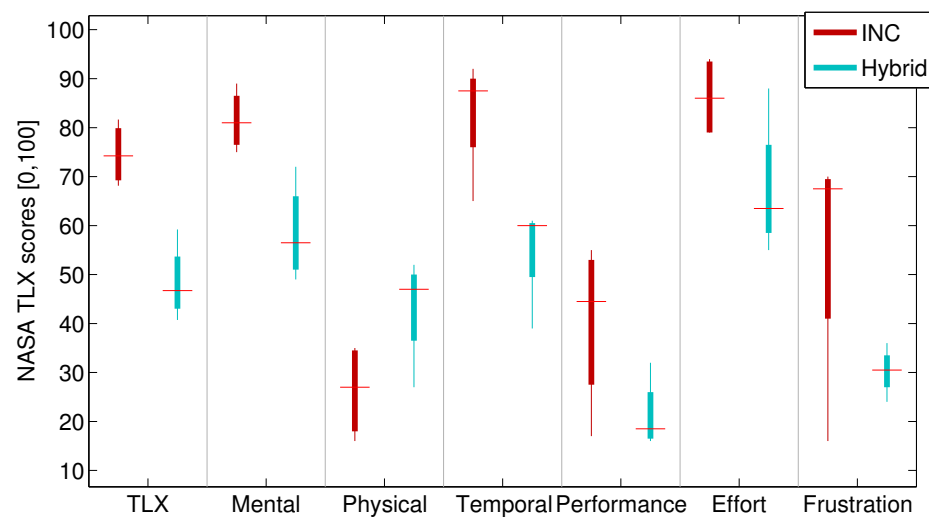

(c) NASA TLX scores

Fig. 5: The error in the duration of the (a) short and (b) long pauses for both control paradigms, for each subject (s1-s4). Positive values indicate that they remained stationary too long, whereas negative values mean that they moved on too soon. (c) The NASA TLX [24] indicates that task load is decreased overall when using the hybrid approach, compared with the original BCI paradigm, which relied heavily on intentional non-control (note: low scores are desirable for every factor, i.e. performance $=0$, implies perfect performance).

\section{REFERENCES}

[1] J. d. R. Millan, F. Renkens, J. Mouriño, and W. Gerstner, "Noninvasive brain-actuated control of a mobile robot by human EEG," IEEE Trans. Biomed. Eng., vol. 51, no. 6, pp. 1026-1033, 2004.

[2] F. Galán, M. Nuttin, E. Lew, P. W. Ferrez, G. Vanacker, J. Philips, and J. d. R Millán, "A brain-actuated wheelchair: Asynchronous and noninvasive brain-computer interfaces for continuous control of robots." Clin. Neurophysiol., vol. 119, no. 9, pp. 2159-2169, Sep 2008.

[3] R. Leeb, S. Perdikis, L. Tonin, A. Biasiucci, M. Tavella, A. Molina, A. Al-Khodairy, T. Carlson, and J. d. R. Millán, "Transferring braincomputer interface skills: from simple BCI training to successful application control," Artificial Intell. in Medicine, 2013, accepted.

[4] J. M. Carmena, M. A. Lebedev, R. E. Crist, J. E. O'Doherty, D. M. Santucci, D. F. Dimitrov, P. G. Patil, C. S. Henriquez, and M. A. L. Nicolelis, "Learning to control a brain-machine interface for reaching and grasping by primates." PLoS Biol., vol. 1, no. 2, p. E42, Nov 2003.

[5] M. Velliste, S. Perel, M. C. Spalding, A. S. Whitford, and A. B. Schwartz, "Cortical control of a prosthetic arm for self-feeding." Nature, vol. 453, no. 7198, pp. 1098-1101, Jun 2008.

[6] G. Müller-Putz, R. Scherer, G. Pfurtscheller, and R. Rupp, "EEG-based neuroprosthesis control: A step towards clinical practice," Neurosci. Lett., vol. 382, pp. 169-174, 2005.

[7] M. Tavella, R. Leeb, R. Rupp, and J. d. R. Millán, "Towards natural non-invasive hand neuroprostheses for daily living," in Proc. 32rd Annu. Int. Conf. IEEE Eng. Medicine and Biology Soc. EMBC 2010, 2010, pp. 126-129.

[8] L. Tonin, T. Carlson, R. Leeb, and J. d. R. Millán, "Brain-controlled telepresence robot by motor-disabled people," in Proc. 32rd Annu. Int. Conf. IEEE Eng. Medicine and Biology Soc. EMBC 2011, 2011, pp. 4227-4230.

[9] T. Carlson and J. d. R. Millán, "Brain-controlled wheelchairs: A robotic architecture," IEEE Robotics and Automation Mag., vol. 20, no. 1, pp. 65-73, 2013.

[10] B. Rebsamen, C. L. Teo, Q. Zeng, M. Ang, E. Burdet, C. Guan, H. Zhang, and C. Laugier, "Controlling a Wheelchair Indoors using Thought," IEEE Intell. Syst., vol. 22, no. 2, pp. 18-24, 2007.

[11] I. Iturrate, J. Antelis, A. Kübler, and J. Minguez, "A noninvasive brainactuated wheelchair based on a P300 neurophysiological protocol and automated navigation," IEEE Trans. Robotics, vol. 25 , no. 3, pp. 614627, june 2009.

[12] T. Carlson and Y. Demiris, "Collaborative control for a robotic wheelchair: Evaluation of performance, attention, and workload," IEEE Trans. Syst. Man Cybern. B, Cybern., vol. PP, no. 99, pp. 1-13, 2012 .
[13] O. Flemisch, A. Adams, S. Conway, K. Goodrich, M. Palmer, and P. Schutte, "The H-Metaphor as a guideline for vehicle automation and interaction," NASA, Tech. Rep. NASA/TM-2003-212672, 2003.

[14] D. Vanhooydonck, E. Demeester, M. Nuttin, and H. Van Brussel, "Shared control for intelligent wheelchairs: An implicit estimation of the user intention," in Proc. 1st Int. Workshop Advances in Service Robot., 2003, pp. 176-182.

[15] T. Carlson and Y. Demiris, "Human-wheelchair collaboration through prediction of intention and adaptive assistance," in Proc. the IEEE Int. Conf. on Robotics and Automation (ICRA), Pasadena, CA, 2008, pp. 3926-3931.

[16] L. Tonin, R. Leeb, M. Tavella, S. Perdikis, and J. d. R. Millán, "The role of shared-control in BCI-based telepresence," in Proc. 2010 IEEE Int. Conf. Syst., Man and Cybern., 2010, pp. 1462-1466.

[17] T. Carlson, L. Tonin, R. Leeb, M. Rohm, R. Rupp, A. Al-Khodairy, and J. d. R. Millán, "BCI Telepresence: A Six Patient Evaluation," in Proc. TOBI Workshop III: Bringing BCIs to End-Users: Facing the Challenge, 2012, pp. 18-19.

[18] J. d. R. Millan, P. W. Ferrez, F. Galán, E. Lew, and R. Chavarriaga, "Non-invasive brain-machine interaction," Int. J. Pattern Recognition and Artificial Intell., vol. 22, no. 5, pp. 959-972, 2008

[19] K. M. Tsui, A. Norton, D. Brooks, H. A. Yanco, and D. Kontak, "Designing telepresence robot systems for use by people with special needs," in Proc. Int. Symp. Quality of Life Technologies 2011: Intell. Syst. for Better Living, held in conjunction with RESNA 2011, Toronto, Canada, June 2011

[20] G. Pfurtscheller, B. Allison, G. Bauernfeind, C. Brunner, T. Solis Escalante, R. Scherer, T. Zander, G. Müller-Putz, C. Neuper, and N. Birbaumer, "The hybrid BCI," Front. Neurosci., vol. 4, p. 42, 2010.

[21] S. Perdikis, A. Ramsey, R. Leeb, J. Williamson, A. Al-Khodairy, R. Murray-Smith, and J. d. R. Millán, "Clinical evaluation of a hybridBCI text-entry system," in Proc. TOBI Workshop III: Bringing BCIs to End-Users: Facing the Challenge, 2012, pp. 75-76.

[22] F. Galán, P. W. Ferrez, F. Oliva, J. Guàrdia, and J. d. R. Millán, "Feature extraction for multi-class BCI using canonical variates analysis," in IEEE Int. Symp. Intelligent Signal Processing, 2007.

[23] S. Perdikis, H. Bayati, R. Leeb, and J. d. R. Millan, "Evidence accumulation in asynchronous BCI," Int. J. Bioelectromagnetism, vol. 13, no. 3, pp. 131-132, 2011.

[24] S. Hart and L. Staveland, "Development of NASA-TLX (task load index): Results of empirical and theoretical research," in Human Mental Workload, P. Hancock and N. Meshkati, Eds. North-Holland, 1988, pp. 139-83. 